\title{
Le Lehrstück brechtien, théâtre de l'accident
}

\section{Sylvain Diaz}

\section{(2) OpenEdition}

\section{Journals}

\section{Édition électronique}

URL : https://journals.openedition.org/agon/1080

DOI : 10.4000 /agon. 1080

ISSN : 1961-8581

\section{Éditeur}

Association Agôn

\section{Référence électronique}

Sylvain Diaz, « Le Lehrstück brechtien, théâtre de l'accident », Agôn [En ligne], 2 | 2009, mis en ligne le 15 décembre 2009, consulté le 14 juillet 2022. URL : http://journals.openedition.org/agon/1080 ; DOI : https://doi.org/10.4000/agon.1080

Ce document a été généré automatiquement le 14 juillet 2022.

Tous droits réservés 


\title{
Le Lehrstück brechtien, théâtre de l'accident
}

\author{
Sylvain Diaz
}

1 «Pour que le théâtre advienne, il faut qu'il y ait quelque chose qui ne va pas ». Ainsi s'exprimait il y a quelques années, à l'occasion d'une conférence intitulée "Théâtre et bonheur » (2007), Michel Vinaver, l'un des auteurs dramatiques français les plus importants de la scène contemporaine. Il poursuit son propos par une longue énumération :

[Pour que le théâtre advienne,] il faut qu'il y ait une catastrophe, un crime, une promesse non tenue, une passion contrariée, un conflit, une offense, un déni de justice, un malentendu, un abus de pouvoir, une attente déçue, la violation d'un interdit, un travers, un accident, un déficit, une trahison, un reniement, une exclusion, une tromperie, une machination, un empêchement... ${ }^{1}$

2 "Accroc dans le tissu de la vie », " rugosité dans la surface plane du réel », l'accident, qui se trouve convoqué au cours de cette liste de termes désignant tous un événement perturbateur, constitue incontestablement, dans la pensée vinavérienne, « la condition nécessaire d'une action dramatique $»^{2}$.

3 Cette conception du théâtre n'a, pour autant, rien d'innovant. Aristote qui, dans La Poétique, définit la tragédie comme "représentation d'action " (mimèsis praxéos) ${ }^{3}$ s'attachait déjà à penser la nécessité de l'événement perturbateur lorsqu'il théorisait le coup de théâtre (péripétéia) comme un " renversement qui inverse l'effet des actions, et ce, suivant notre formule, vraisemblablement ou nécessairement ${ }^{4}$. Les auteurs classiques français, qui élaborent leur esthétique à partir de la Poétique aristotélicienne qu'ils lisent et commentent abondamment, la considérant comme un modèle à adopter, ne sont pas en reste : ainsi l'Abbé d'Aubignac, l'un de ses principaux théoriciens, peut-il penser la tragédie française comme la représentation « réglée » d'un " dérèglement " 5 . Assurément, l'événement perturbateur - qu'on le nomme «coup de théâtre», «dérèglement " ou encore "accident» - constitue depuis l'origine du théâtre le fondement de toute représentation dramatique, et ce même chez les auteurs qui, tels Maurice Maeterlinck qui revendiquera l'invention d'un "Théâtre statique " ${ }^{6}$, tâcheront de renoncer au principe d'action: en effet, Les Aveugles (1890), pièce exposant une 
situation parfaitement immobile, trouve encore son origine dans une "turbulence $»^{7}$ pour reprendre un autre terme de la poétique vinavérienne, puisqu'un groupe d'aveugles se trouve immobilisé dans une forêt à la suite de la mort brutale et inattendue de leur guide. Il apparaitt toutefois que, sur la scène moderne, l'accident est progressivement vidé de sa valeur dynamique en tant qu'il ne participe plus du développement d'une action. Aussi, dans le prolongement de Maeterlinck, Ibsen, Strindberg ou encore Tchekhov, d'autres auteurs, parmi lesquels le dramaturge allemand Bertolt Brecht, tenteront-ils au début du XXe siècle d'aller plus loin et de rompre avec l'accident.

4 À l'origine du théâtre brechtien, ce constat formulé sur le ton de l'évidence dans l'un des premiers textes théoriques importants du dramaturge allemand, «Sur une nouvelle dramaturgie » (1928) : «La vieille forme du drame ne permet pas de représenter le monde tel que nous le voyons aujourd'hui $»^{8}$. C'est donc en opposition au théatre "dramatique », ce " théâtre du passé ", que Brecht travaille à l'élaboration d'une forme théâtrale nouvelle et actuelle qualifiée d' "épique »". Celle-ci se démarque en tant qu'elle entend porter à la scène non «l'extraordinaire » comme c'est l'usage dans la tragédie mais bien "l'ordinaire», "le quotidien», explique le personnage du Philosophe dans L'Achat du cuivre (1939-1941), grand œuvre théorique de Brecht qui se présente sous une forme dialoguée. Ce resserrement du théâtre autour de 《l'expérience journalière $»^{10}$ pour reprendre la formule diderotienne ne va toutefois pas sans poser problème puisque, explique le Comédien, «la seule réalité qu'éveille en nous la quotidienneté est celle de l'ennui ${ }^{11}$. Ceci suppose de ce fait de ne pas rompre avec l'événement perturbateur, avec l'accident, ainsi que l'envisageait Brecht initialement, mais d'en développer une nouvelle approche, ce qui est notamment à l'œuvre dans son théâtre didactique composé de six pièces rédigées entre 1929 et 1934 : Le Vol au-dessus de l'océan (1929), L'Importance d'être d'accord (1929), Celui qui dit oui Celui qui dit non (1930), La Décision (1931), L'Exception et la règle (1930) et Les Horaces et les Curiaces (1934).

5 Si elle s'inscrit dans le prolongement des premiers "essais» (Versuche) de théâtre épique - c'est en effet ainsi que Brecht désigne ses premières pièces, tant Dans la jungle des villes (1924) que Homme pour homme (1926) ou encore L'Opéra de quat'sous (1928) -, la pièce didactique (Lehrstück) n'en constitue pas moins une rupture dans l'œuvre de Brecht. La profonde mutation de son écriture, relevée par Bernard Dort dans sa remarquable Lecture de Brecht (1960), en témoigne :

6 En dépit de leur apparence impersonnelle, les Lehrstücke cö̈ncident avec une conversion. Ils s'enracinent dans la volonté de Brecht de devenir communiste. Rompant avec la société bourgeoise qu'il avait attaquée et scandalisée mais qui lui avait accordé sa consécration, Brecht fait de cette rupture un holocauste. Il se sacrifie lui-même; il sacrifie ses propres œuvres. Il sacrifie même une partie de son talent d'écrivain, d'homme de théâtre - celle qui lui avait valu la gloire: ses Lehrstücke sont d'une impitoyable, d'une monastique sécheresse ; ils grincent. À l'abondance, à la prodigalité de L'Opéra de quat'sous succèdent une draconienne économie de vocabulaire, une implacable rigidité de construction. ${ }^{12}$

7 Comprendre l'enjeu de ce théâtre didactique suppose toutefois de revenir, avec Philippe Ivernel, sur le contexte historique de l'écriture du Lehrstück brechtien :

la période historique du Lehrstück correspond à une période cruciale de l'histoire

allemande (dans laquelle elle ne se laisse pas enfermer. Le Lehrstück est toujours à 
venir), celle du choix entre socialisme et barbarie (1929-1933). L'écrivain de théâtre sent alors le sol lui brûler sous les pieds. Il esquisse une sortie hors des lieux officiels où se diffusent des images administrées (les "spectacles ») et tente de gagner l'espace quotidien où s'élabore un nouvel usage de la culture, non pour un nouveau "public », mais avec de nouveaux " usagers ": la jeunesse, le mouvement ouvrier, c'est-à-dire les forces vives intéressées à la transformation sociale, ceux qui ne pensent pas sans motif ni conséquence. Tous ceux-là sont invités à jouer plutôt qu'à voir, comme à faire plutôt qu'à sentir : en résumé à pratiquer le théâtre, et ceci comme une école de pratique. ${ }^{13}$

8 Le Lehrstück est donc, en premier lieu, un théâtre destiné à la pratique et, particulièrement, à la pratique amateur. De fait, Brecht n'aura de cesse de l'affirmer, révélant par là même le mode singulier de représentation de ces textes : "la pièce didactique enseigne $d u$ fait qu'elle est jouée, non du fait qu'elle est vue. En principe aucun spectateur n'est nécessaire à la pièce didactique ${ }^{14}$. L'enjeu du Lehrstück est en effet de confronter les acteurs à des situations problématiques exigeant une solution pratique. Or, ces situations problématiques trouvent le plus souvent leur source dans un accident, ainsi qu'en témoigne L'Importance d'être d'accord, pièce sur laquelle se concentrera notre analyse. Le Lehrstück brechtien se donne dès lors à comprendre comme un espace d'expérimentation privilégié où tenter de penser de manière nouvelle le rapport entre théâtre et accident.

\section{L'accident comme processus d'expérimentation}

Dans Le Vol au-dessus de l'océan, son premier Lehrstück qui a pour singularité d'avoir été écrit pour la radio, Brecht propose à la communauté théâtrale de «refaire l'expérience ${ }^{15}$ de la première traversée aérienne de l'océan Atlantique effectuée en mai 1927 par Charles Lindbergh auquel est substitué un personnage choral et anonyme $^{16}$. Si l'exploit technique est couronné de succès dans le premier Lehrstück, il est à l'inverse mis en échec dans L'Importance d'être d'accord, version négative du Vol audessus de l'océan ${ }^{17}$. Ainsi assiste-t-on, dès le deuxième tableau, à la chute d'un groupe d'aviateurs qui procédaient, à leur tour, à une traversée aérienne de l'océan Atlantique : «LE CHEF DU CHCUR, s'adressant aux aviateurs tombés: Vous ne volerez plus à présent. / Inutile désormais d'aller toujours plus vite. / À même le sol / C'est assez haut / Pour vous. / Vous gisez immobiles / Cela suffit. / [...] Ils ont / Explosé dans les airs, sont / Tombés à terre et / Ne veulent pas mourir $»^{18}$.

10 Si l'accident brechtien évoque le "dérèglement » de la dramaturgie classique en tant qu'il vient perturber la situation initiale, on ne saurait toutefois manquer de remarquer que, dans L'Importance d'être d'accord, la chute des aviateurs ne provoque aucun renversement de l'action. Dès la fin du second tableau, le chœur, puis le récitant orientent en effet la pièce vers l'examen scientifique d'un problème donné :

Les Aviateurs tombés. Nous vous en prions,

Faites un pas vers nous,

Et donnez-nous à boire,

Et mettez un coussin sous notre tête,

Et venez-nous en aide, car

Nous ne voulons pas mourir.

Le Chœur, s'adressant à la foule. Vous entendez, quatre hommes

Demandent votre aide. [...]

Répondez-nous : 
Faut-il leur venir en aide?

La Foule, répondant au Chœur. Oui.

Le Chœur, à la foule. Vous ont-ils aidé, vous?

La Foule. Non.

Le Récitant, s'adressant à la foule. Par-dessus ces corps qui refroidissent, nous allons

examiner

S'il est d'usage que l'homme vienne en aide à l'homme.

11 L'Importance d'être d'accord l'illustre: dans le prolongement des auteurs modernes, Brecht refuse à l'accident toute valeur dynamique que reconnaissaient, à l'inverse, les Classiques français au « dérèglement ». Ce qui l'intéresse dans l'accident n'est pas tant le déséquilibre produit que le nouvel équilibre établi qu'il se propose d'étudier scientifiquement : «Les Lehrstücke brechtiens, écrit Philippe Ivernel, confirment à leur manière qu'écrire pour le théâtre, c'est penser une idée jusqu'au bout, selon le mot de Kaiser. Une thèse (une situation, un comportement) étant posée au départ, elle sera soumise, par répétition et variation, à un processus d'expérimentation et d'autoapprentissage méthodique $»^{19}$.

L'Importance d'être d'accord offre une illustration précise de ce «processus d'expérimentation ». Ayant mis au jour, au second tableau, l'objet de sa pièce, Brecht mène, au troisième tableau, une série d' « Enquêtes [Untersuchungen] » ayant pour enjeu d'établir «si l'homme est une aide pour l'homme " ${ }^{20}$. Dans la première enquête où dialoguent le Chœur et le Chef du Chœur, les personnages s'interrogent pour savoir si la science est profitable à l'homme :

LE CHEF DU CHEUR, s'avance. L'un de nous a traversé la mer, il

A découvert un nouveau continent.

Mais à sa suite d'autres sont allés là-bas

Construire de grandes villes à force

De sueur et d'intelligence.

LE CHCUR, répondant. Le pain n'est pas devenu moins cher pour autant.

LE CHEF DU CHCUR. L'un de nous a construit une machine

Grâce à quoi la vapeur actionne une roue. Ce fut

La mère de bien des machines.

Mais des hommes y travaillent

Jour après jour.

LE CHÆUR, répondant. Le pain n'est pas devenu moins cher pour autant.

LE CHEF DE CHÆUR. Beaucoup d'entre nous ont réfléchi

Sur la rotation de la terre autour du soleil, sur

Le cœur de l'homme, les lois

Universelles, la composition de l'air

Et le poisson des mers profondes.

Et ils ont découvert

De grandes choses

LE CHÆUR, répondant. Le pain n'est pas devenu moins cher pour autant.

$\mathrm{Au}$ contraire, La misère s'est accrue dans nos villes

Et depuis longtemps déjà

Ce qu'est un homme, personne ne le sait plus.

Ainsi, tandis que vous voliez, rampait

$\mathrm{Au}$ sol un être semblable à vous

Et qui n'avait plus rien d'un homme ! ${ }^{21}$

13 Le Chef du Chœur demande alors à la Foule, personnage collectif engageant nécessairement le spectateur dans la représentation, de tirer une conclusion de cette 
première enquête : «LE CHEF DU CHEUR, s'adressant à la foule: Donc, l'homme est-il une aide pour l'homme? LA FOULE répond: Non $»^{22}$.

La deuxième enquête, très brève, relève, elle, du théâtre documentaire initié par Erwin Piscator, metteur en scène allemand avec lequel le jeune Brecht collabora au cours des années 1920. Ce théâtre se distingue notamment par l'exploitation de moyens techniques nouveaux - projections photographiques ou cinématographiques, tapis roulants, scènes tournantes... - rendant possible une représentation inédite de la réalité23. Si Brecht s'est souvent montré critique, voire condescendant à l'égard de Piscator qu'il désignait comme "l'auteur de spectacles» et dont il entendait se distinguer en se nommant "l'auteur de pièces ${ }^{24}$, le troisième tableau de L'Importance d'être d'accord atteste toutefois du réinvestissement, dans le Lehrstück, de certains procédés du théâtre documentaire. La seconde enquête visant à déterminer si l'homme est une aide pour l'homme procède ainsi par projection photographique :

LE CHEF DU CHCEUR, s'adressant à la foule. Regardez ces photographies ; direz-vous après

cela

Que l'homme est une aide pour l'homme?

On présente vingt photographies qui montrent comment de nos jours des hommes sont massacrés par d'autres hommes.

LA FOULE crie. L'homme n'est pas une aide pour l'homme ${ }^{25}$.

Dans la troisième enquête, sans doute la plus longue, Brecht met enfin en scène un numéro de clowns : " LE CHEF DU CHœUR, s'adressant à la foule: Regardez ces numéros de clown. Ils vont vous montrer / Comment des hommes viennent en aide à un homme! Trois clowns de cirque, dont l'un, nommé monsieur Schmitt, est un géant, montent sur l'estrade $»^{26}$. Au fil de la scène, les Clowns $n^{\circ} 1$ et $n^{\circ} 2$ se proposent de venir en aide à monsieur Schmitt qui dit souffrir de divers maux :

MONSIEUR SCHMITT. Dites-moi, j'ai presque l'impression que mon pied gauche me fait

un peu mal.

$\mathrm{N}^{\circ} 1$. Très mal?

MONSIEUR SCHMITT, douloureusement. Comment?

$\mathrm{N}^{\circ} 1$. Est-ce qu'il vous fait très mal?

MONSIEUR SCHMITT. Oui, il commence à me faire très mal...

$\mathrm{N}^{\circ}$ 2. Parce que vous restez debout.

MONSIEUR SCHMITT. Alors, je dois m'asseoir?

$\mathrm{N}^{\circ} 1$. Non, surtout pas, il faut éviter ça à tout prix.

$\mathrm{N}^{\circ}$ 2. Si le pied gauche vous fait mal, il n'y a qu'une chose à faire : enlever le pied

gauche.

$\mathrm{N}^{\circ} 1$. Et le plus vite sera le mieux.

MONSIEUR SCHMITT. Ma foi, si vous croyez...

$\mathrm{N}^{\circ}$ 2. Bien sûr.

Ils lui scient le pied gauche. ${ }^{27}$

Le démembrement progressif de monsieur Schmitt que Brecht met en scène - les deux clowns, hilares, lui coupent successivement la jambe droite, l'oreille gauche, le bras gauche et la moitié supérieure du crâne ${ }^{28}$ - se referme sur ce commentaire ironique :

Les Clowns dévissent la tête de monsieur Schmitt qui tombe à la renverse.

MONSIEUR SCHMITT. Hé là ! Quelqu'un! Mettez-moi la main sur le front.

$\mathrm{N}^{\circ} 1$. Où ça?

MONSIEUR SCHMITT. Prenez-moi par la main.

$\mathrm{N}^{\circ} 1$. Où ça?

$\mathrm{N}^{\circ} 2$. Vous sentez-vous plus léger maintenant, monsieur Schmitt?

MONSIEUR SCHMITT. Non. Je suis couché le dos sur un caillou. 
$\mathrm{N}^{\circ}$ 2. Ah! Monsieur Schmitt, on ne peut pas tout avoir.

Tous deux rient aux éclats. Fin du numéro de clowns. ${ }^{29}$ que cette séquence traumatisante pour le spectateur, où se rejoue d'une certaine manière l'accident des personnages, nécessite une interprétation verbale afin d'interdire toute sidération ${ }^{37}$. Aussi le tableau exclusivement visuel de la " Contemplation des morts » est-il suivi d'un tableau verbal intitulé «Lecture des textes du commentaire » ayant pour fonction première de l'interpréter, et par là même d'élucider la fable. Le Récitant propose ainsi aux Aviateurs abattus la lecture d'un livre ayant valeur d'enseignement au moment même de leur mort: "LE CHÆUR RÉGULIER, aux aviateurs abattus. Nous ne pouvons rien pour vous. / Il n'est qu'un conseil, / Qu'une attitude / Que nous puissions vous proposer. / Mourez, mais apprenez, / Apprenez, mais n'apprenez pas de travers $\gg .^{38}$

Cet enseignement se fait en quatre étapes. La première est une invitation à faire l'expérience du «renoncement » : «Celui qui arrache une chose la tiendra. Celui à qui 
on arrache une chose, lui aussi la tiendra. Mais celui qui tient une chose, on la lui arrachera. [...] Mais comment, sans entraînement, soulever une pierre? Comment soulever une grosse pierre? Comment, si l'on ne s'est pas entraîné au renoncement, abandonner sa table? Comment abandonner tout ce que l'on a et ce que l'on n'a pas? $»^{39}$. La seconde étape est un encouragement à faire l'expérience de "la plus petite dimension de [soi]-même » (in seiner kleinsten Größe) : "Quand le philosophe fut pris dans une grande tourmente, il se trouvait assis dans une grande voiture et il tenait beaucoup de place. La première chose qu'il fit fut de descendre de la voiture, la seconde d'enlever son manteau, la troisième de s'étendre par terre. Il surmontait ainsi la tourmente dans la plus petite dimension de lui-même $»^{40}$. Si la troisième étape est de nouveau encouragement à l'abandon, la dernière étape est, elle, enseignement de l'«accord» (Einverständnis) : «Si le philosophe a surmonté la tourmente, c'est qu'il connaissait la tourmente, qu'il était d'accord avec elle. Donc, si vous voulez surmonter la mort, il faut la connaître et être d'accord avec elle $»^{41}$.

Cet enseignement constitue ainsi un commentaire non seulement du tableau précédent mais de la pièce elle-même puisqu'il en révèle l'enjeu: la mort à laquelle sont confrontés les personnages - mais aussi les spectateurs du fait des projections photographiques - n'est autre qu'une épreuve au cours de laquelle ils sont invités à consentir à l'abandon, à faire l'expérience de la plus petite dimension d'eux-mêmes, à marquer leur accord avec le monde. Mais cette expérience, loin d'être exclusivement macabre, est également politique. Si, dans Le Vol au-dessus de l'océan, Brecht vantait le progrès technique en tant qu'il participe d'une « combat contre l'anachronique ${ }^{42}$, il se voit au contraire condamné dans L'Importance d'être d'accord en tant que les Aviateurs y ont sacrifié toute identité et donc toute conviction :

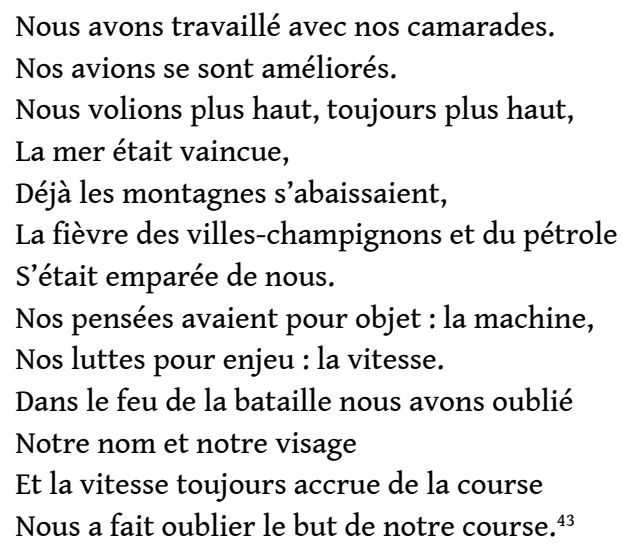

La première enquête du troisième tableau entend dès lors démontrer que ce qui fait défaut au progrès technique dans L'Importance d'être d'accord, c'est, selon les propos du Chœur, une implication sociale. Car, si «Beaucoup d'entre nous ont réfléchi / Sur la rotation de la terre autour du soleil, sur / Le cœur de l'homme, les lois / Universelles, la composition de l'air / Et le poisson des mers profondes ", cela n'a pas changé la vie des hommes : «Au contraire, remarque le Chœur, La misère s'est accrue dans nos villes » ${ }^{44}$. La référence à Galilée qui ouvre ce texte, pour être discrète, n'en est pas moins décisive. Près de vingt ans plus tard, alors qu'il travaille à une nouvelle version de La Vie de Galilée (1955), Brecht, hanté par les bombardements atomiques d'Hiroshima et Nagasaki, condamnera le «crime » de son personnage éponyme, n'ayant reconnu au progrès de la connaissance auquel il participait aucune implication sociale : 
En réalité, Galilée a enrichi l'astronomie et la physique en privant en même temps ces sciences d'une grande partie de leur signification sociale. [...]

Le crime de Galilée peut être considéré comme le "péché originel » des sciences modernes de la nature. De la nouvelle astronomie, qui intéressait très profondément une nouvelle classe, la bourgeoisie, car elle apportait son concours aux courants sociaux révolutionnaires de l'époque, il fit une science particulière strictement limitée qui, certes, précisément du fait de sa "pureté ", c'est-à-dire de son indifférence au mode de production, pouvait se développer tranquillement, toute proportion gardée.

La bombe atomique est comme phénomène aussi bien technique que social le produit final typique de sa prestation scientifique et de sa carence sociale. ${ }^{45}$

25 À l'inverse de Galilée, les Aviateurs de L'Importance d'être d'accord
le Chœur à renouer avec le monde, à se réinscrire dans le monde :

Vous qui êtes d'accord avec le cours des choses

Ne retournez pas au néant.

Ne vous dissolvez pas comme le sel dans l'eau, mais au contraire

Levez la tête.

Mourez votre mort

Comme vous avez œuvré votre œuvre,

Accomplissez une révolution.

Donc, au moment de mourir

Ne vous conformez pas à la mort.

Acceptez la mission que nous vous confions :

Reconstruisez notre avion.

Mettez-vous à l'ouvrage!

Afin de voler pour nous

À l'endroit où nous avons besoin de vous

À l'heure où c'est nécessaire. ${ }^{46}$ 
de hâte, mais de les révéler, mieux de les susciter patiemment, méthodiquement, afin d'en tester la productivité. D'où la place faite à l'accident, que d'une certaine manière il installe au cœur de son fonctionnement $»^{52}$. Résolument, le Lehrstück brechtien est théâtre de l'accident, cet « autre nom pour le concret " ${ }^{53}$, conclut l'essayiste.

Cette prégnance de l'accident dans le théâtre brechtien se trouvera consacrée dans le discours théorique dès 1935 avec la rédaction d'un poème composé pour L'Achat du cuivre qui encourage les "Artistes » à rechercher «le théâtre quotidien, multiple et sans gloire, / Mais si vivant, terrestre, nourri de la vie sociale des hommes / Et qui se joue dans la rue »: "Voyez cet homme, au coin de la rue! Il montre comment / L'accident s'est déroulé. Le voici en train / De soumettre le conducteur au jugement de la foule. Il le montre / Assis au volant ; et maintenant / Il imite celui qui a été écrasé, apparemment / Un vieil homme ${ }^{54}$. Ainsi la «scène de la rue» et, plus particulièrement, la scène d'accident constitue-t-elle, dans la pensée brechtienne, le "modèle type d'une scène de théâtre épique ", ainsi qu'il est affirmé dans un texte non plus poétique mais bien synthétique composé en 1938 à l'intention de comédiens :

Il est relativement aisé de proposer un modèle type de théâtre épique. Lors des exercices pratiques, j'avais l'habitude de choisir pour exemple d'un théâtre épique des plus simples, en quelque sorte "naturel», un processus susceptible de se dérouler à n'importe quel coin de rue : le témoin oculaire d'un accident montre, gestes à l'appui, à des gens attroupés, comment les choses se sont passées. Ces gens peuvent n'avoir rien vu ou simplement n'être pas de l'avis du témoin, voir les choses "autrement»; l'essentiel est que le démonstrateur montre le comportement du conducteur, ou de la victime, ou de l'un et de l'autre, de manière telle que l'assistance puisse se faire une opinion sur l'accident. ${ }^{55}$

À partir du Lehrstück, Brecht s'est ainsi attaché à développer dans son œuvre une approche nouvelle et, pour tout dire, novatrice de l'accident au théâtre : vidé de toute valeur dynamique, il constitue désormais le cadre d'un procès du monde où, tour à tour, « agents » et « patients » de l'action pour reprendre les termes de Paul Ricœur ${ }^{56}$ portent témoignage de l'événement auquel ils ont participé ou assisté et méditent ensemble sur ses causes et conséquences afin de déterminer comment agir à l'avenir. En tant que tel, l'accident participe de manière essentielle d'une détermination critique du théâtre brechtien, offrant la possibilité aux acteurs comme aux spectateurs d'envisager à nouveaux frais la réalité ainsi que les moyens de la transformer.

\section{Épilogue - Du « Temps-Accident » dans le théâtre d'Edward Bond}

31 Il convient, en épilogue à cet article, de tenter de prendre la mesure de la portée de cette nouvelle approche de l'accident initiée par Brecht dans les dramaturgies qui lui sont ultérieures et, particulièrement, dans les dramaturgies contemporaines. L'œuvre d'Edward Bond semble se prêter à une telle confrontation. Parce que, dès la fin des années 1970, il qualifie son théâtre d' « épique $»^{57}$, le dramaturge britannique s'inscrit en effet dans le prolongement de Brecht dont il reconnaît volontiers l'influence, ce que pourrait confirmer ces propos rapportés par David Hirst en 1985 :

Brecht était un expérimentateur, un exploreur mais il ne répondait pas à toutes nos questions. Le temps qui s'est écoulé entre sa mort et aujourd'hui nous a conféré plus d'expérience, nous a transmis plus d'histoire à retranscrire. La tragédie du drame du XX $\mathrm{XX}^{\mathrm{e}}$ siècle, c'est que Brecht soit mort avant d'avoir pu achever sa dernière 
série de pièces, pièces qu'il aurait pu écrire en tant que membre et que travailleur d'une société marxiste. La perte est sévère. Aussi nous revient-il d'écrire les pièces qu'il n'a pas écrites. ${ }^{58}$ Rach constitue néanmoins une tentative d'explication de cette condamnation brutale si ce n'est de Brecht, du moins de la distanciation brechtienne, sans pour autant la remettre en cause :

J'ai qualifié le théâtre fondé sur l'effet-D (effet de distanciation) de Théâtre d'Auschwitz. Il est évident que je n'emploie pas cette expression au premier degré. Prise au premier degré elle est le contraire de la vérité. À Auschwitz les nazis n'auraient pas joué Brecht, ils l'auraient exterminé. Brecht a consacré sa vie et son énergie à tenter d'empêcher que des cercles de l'enfer tels qu'Auschwitz - ou le Goulag - soient possibles. On ne saurait rien dire de plus sérieux sur Brecht que ce que j'ai dit dans les « Notes sur Café » (pour le Théâtre National de la Colline).63

À partir de l'exemple de La Vie de Galilée, Edward Bond tente dès lors d'établir que l'œuvre de Brecht ne saurait constituer " un théâtre total capable de décrire nos vies et de nous faire accéder à l'acte de création de soi ${ }^{64}$. Aussi, pour pouvoir " écrire sur [notre] époque ", faut-il aux auteurs dramatiques contemporains, à l'instar de Sarah Kane, «non pas rejeter Brecht mais alléguer qu'il appartient à un paradigme de la connaissance désormais dépassé $\aleph^{65}$. De fait, dès 1991, Edward Bond entendait « construire un théâtre post-brechtien qui s'appuie sur les fondations de l'œuvre de Brecht mais incorpore aussi le post-modernisme, en évitant de se réfugier dans le théâtre de Robert Wilson et Heiner Müller ${ }^{66}$. Or, ce dépassement de l'œuvre brechtienne se donne particulièrement à lire dans la théorisation bondienne de l'accident. 

ses textes théoriques, l'événement théâtral :

Une pièce de théâtre ne montre pas des événements mais le sens de ces événements. Les valeurs nous servent à émettre des jugements. Si un accident se produit dans la vie réelle, nous essaierons peut-être d'établir les faits. Mais un juge, un juré et un témoin auront chacun une version différente de l'accident, fondée sur l'attitude sociale qui est la leur face aux accidents et à tous les autres événements. Il est impossible d'établir même les faits les plus essentiels sans avoir recours à des jugements de valeur. Émettre un jugement est aussi complexe sur scène que dans la vie. ${ }^{67}$

En recourant à la métaphore accidentelle pour définir l'événement théâtral, Edward Bond s'inscrit résolument dans le prolongement de Brecht. Il convient toutefois de remarquer que la description de la scène d'accident dans la théorie bondienne procède d'une inversion essentielle de la perspective brechtienne. En effet, si le dramaturge allemand organise rigoureusement sa théorisation de la « scène de la rue » à partir du point de vue du témoin, le dramaturge britannique l'organise, lui, à partir du point de vue de la victime : "Lorsque des gens relatent quelle impression cela fait de se trouver dans un grave accident, ils disent avoir gardé leur calme et eu l'impression que le temps se ralentissait, si bien qu'ils remarquaient des choses qu'ils n'auraient pas eu le temps de remarquer s'ils étaient simplement passés par là $\aleph^{68}$. Aussi Edward Bond se proposet-il, dans ses pièces, de porter l'accident lui-même à la scène, ainsi qu'il l'explique à partir d'une référence aux Trois Soeurs (1900) de Tchekhov, pièce qu'il a traduite, en 1967, pour le Royal Court Theater de Londres :

Vers la fin des Trois Sceurs de Tchekhov, les personnages qui sont en scène parlent ou se taisent tandis qu'au dehors leur société - se servant de Solioni comme exécutant - commet un meurtre. Dans notre théâtre, la violence a lieu sur scène parce que c'est là qu'elle se situe dans la vie que nous menons: elle n'attend pas dans les coulisses comme elle le faisait chez Tchekhov. Ses personnages mènent une enquête sur un accident qui n'a pas encore eu lieu mais qu'ils ne peuvent empêcher. Notre scène à nous est le théâtre même de l'accident. Tout se déroule donc plus vite et de façon plus intense que chez Tchekhov : tout se passe en temps-accident. Or c'est parce que les choses se produisent si rapidement qu'il est difficile de les empêcher ou de leur échapper. ${ }^{69}$

Il apparaît ainsi que l'accident ne désigne pas seulement, dans le discours théorique bondien, un processus événementiel mais également une temporalité singulière, frappée d'étrangeté en ceci qu'elle nous fait pénétrer « dans un monde où nous voyons et comprenons différemment $»^{70}$, ainsi que l'explique le dramaturge britannique dans une lettre à Antje Lenkeit datée de 1997. Le «temps-accident " n'est autre en ce sens qu'un " phénomène existentiel $»^{71}$, note Georges Bas. La théorisation bondienne de l'accident témoignerait en ce sens d'une tentative de conciliation inédite de sa valeur dynamique héritée du "théâtre du passé " pour reprendre une fois encore la formule brechtienne et de sa portée critique initiée par le dramaturge allemand. Or, les pièces les plus récentes d'Edward Bond témoignent incontestablement d'un dépassement de l'un par l'autre : l'accident a avant tout pour fonction, dans la Tétralogie de la Colline ${ }^{72}$, de confronter acteurs et spectateurs à une situation extrême où l'humain se trouve mis à l'épreuve ${ }^{73}$.

En ce sens, le théâtre de l'accident que revendique Edward Bond est différent et, pour tout dire, opposé à celui élaboré par Brecht dans ses pièces didactiques. C'est que l'accident ne participe plus, dans les pièces du dramaturge britannique, du 
développement d'un commentaire mais bien de celui d'une action. Ce théâtre ne saurait être en ce sens post-brechtien ainsi que l'affirme son auteur ; il serait au contraire prébrechtien en tant qu'il marque, du point de vue de l'accident, un retour à une esthétique antérieure à celle du dramaturge allemand.

Si Brecht « appartient à un paradigme de la connaissance désormais dépassé » ainsi que l'affirme Edward Bond, il convient pour autant de ne pas liquider purement et simplement son héritage ${ }^{74}$ mais de revenir de manière critique sur son théâtre et, particulièrement sur son théâtre didactique trop souvent dénigré, pour tenter d'exploiter cette approche nouvelle et novatrice de l'accident au théâtre qu'il a pensée dans ses textes théoriques et mis en œuvre - mis en acte, devrait-on dire - dans ses pièces.

\section{NOTES}

1. Michel Vinaver, "Théâtre et bonheur", in Lexi / textes 11 - Inédits et commentaires, Paris, L'Arche Éditeur / Théâtre National de la Colline, 2007, p. 247.

2. Ibid., pp. 247-248.

3. Aristote, La Poétique, traduit et présenté par Roselyne Dupont-Roc et Jean Lallot, Paris, Seuil, collection « Poétique », 1980, chapitre 6, 1449 b 24-28, p. 53.

4. Ibid., chapitre 11, 1452 a 22-24, p. 71. Aristote appuie sa définition d'un exemple emprunté à la tragédie de Sophocle, Édipe roi (- 430), érigée en modèle au fil de La Poétique: «dans l'ÆEdipe, quelqu'un vient pour réconforter ⿷匚dipe et le délivrer de ses craintes au sujet de sa mère ; mais, en lui révélant son identité, il fait l'inverse ». Ibid., chapitre 11, 1452 a 24-26, p. 71. Le coup de théâtre aristotélicien fait l'objet d'une analyse précise et lumineuse par Roland Barthes, analyse qui intervient, de manière inattendue, dans un article consacré, non à la tragédie grecque, mais à la pièce de Brecht, Mère Courage et ses enfants (1939) : "La Tragédie se définit par une antinomie rigoureuse et par suite apparemment délibérée entre les faits et les conséquences : vous faites ceci pour vous sauver, et c'est précisément cela qui vous perdra. Le retournement tragique (ce qu'on appelait la péripétie) est un retournement exactement symétrique : pour mourir, il suffira aux enfants de Mère Courage d'accomplir à la lettre la fausse prédiction que leur mère a faite pour les sauver. Dans la tragédie, c'est cette symétrie qui manifeste la nature magique du Destin, car seul un Dieu peut mesurer aussi intelligemment la déviation d'une cause en sa conséquence contraire, seul un Dieu peut connaître l'art d'une symétrie aussi juste ». Roland Barthes, "Sept photos modèles de Mère Courage » (1959) in Écrits sur le théâtre, textes réunis et présentés par JeanLoup Rivière, Paris, Éditions du Seuil, collection « Points / Essais », 2002, p. 263.

5. François Hédelin, Abbé d'Aubignac, La Pratique du théâtre (1657), Paris, Honoré Champion, 2002, p. 68. Sur cette question du "dérèglement", $c f$. également Pierre de Villiers, Entretien sur les tragédies de ce temps (1675), in Racine, Euvres complètes, édition établie par Georges Forestier, Paris, Gallimard, « Bibliothèque de la Pléiade », 1999, volume I (Théâtre - Poésie), p. 793. À propos de ce curieux paradoxe esthétique, on ne manquera pas de consulter le très précieux ouvrage de Georges Forestier, Passions tragiques et règles classiques - Essai sur la tragédie française, Paris, P.U.F., 2003.

6. Maurice Maeterlinck, «Le Tragique quotidien» (1896) in Cuvres I: Le Réveil de l'âme, poésie et essais, édition établie par Paul Gorceix, Bruxelles, Éditions Complexe, 1999, p. 490. À propos du 
«Théâtre statique » de Maeterlinck et son devenir sur la scène contemporaine, $c f$. Hélène Kuntz, Mireille Losco, «Statisme » in Poétique du drame moderne et contemporain - Lexique d'une recherche, dirigé par Jean-Pierre Sarrazac, assisté de Hélène Kuntz, David Lescot, Mireille Losco, Catherine Naugrette, Études théâtrales, nº 22, 2001, p. 120.

7. Michel Vinaver, «J'essaie de faire des formes... », entretien avec Joseph Danan, Évelyne Ertel, Daniel Lemahieu, Catherine Naugrette et Jean-Pierre Ryngaert, in Michel Vinaver, Côté texte / Côté scène, sous la direction de Catherine Naugrette, Registres, Hors-Série ${ }^{\circ}{ }^{1}$, 2008, pp. 46-47.

8. Bertolt Brecht, «Sur une nouvelle dramaturgie » (1928), in Écrits sur le théâtre, édition établie sous la direction de Jean-Marie Valentin, avec la collaboration de Bernard Banoun, Jean-Louis Besson, André Combes, Jeanne Lorang, Francine Maier-Schaeffer et Marielle Silhouette, Paris, Gallimard, collection « Bibliothèque de la Pléiade », 2000, p. 124.

9. L'opposition entre "théâtre épique » et "théâtre dramatique » est notamment formulée dans un tableau publié dans les «Notes sur l'opéra Grandeur et décadence de la ville de Mahagonny " (1930) in Écrits sur le théâtre, volume II, traduits par Jean Tailleur et Édith Winkler, Paris, L'Arche Éditeur, 1979, pp. 328-329. Pour éviter toute confusion avec l'édition de référence des Écrits sur le théâtre publiée dans la collection «Bibliothèque de la Pléiade " aux éditions Gallimard, cette seconde édition sera ainsi désignée par la suite en note: "Écrits sur le théâtre [L'Arche]».

10. Diderot, Entretiens sur Le Fils naturel (1757), in CEuvres, édition établie par Laurent Versini, Paris, Robert Laffont, collection « Bouquins », 1996, volume IV « Esthétique - Théâtre », p. 1132.

11. Bertolt Brecht, L'Achat du cuivre (1939-1941), in Écrits sur le théâtre, op. cit., p. 546.

12. Bernard Dort, Lecture de Brecht (1960), Paris, Éditions du Seuil, collection «Pierres Vives ", 1999 , p. 85.

13. Philippe Ivernel, « Du spectacle au montage ininterrompu : le Lehrstück brechtien », in Collage et montage au théâtre et dans les autres arts durant les années vingt, Lausanne, La Cité - L’Âge d'homme, collection « Théâtre années vingt », 1978, p. 267.

14. Bertolt Brecht, «Sur la théorie de la pièce didactique », in Écrits sur le théâtre [L'Arche], volume II, op. cit., p. 341.

15. Alain Gintzburger, Grégoire Ingold, Philippe Ivernel, «Brecht avec ou sans modèle?Conversation ", entretien avec Jean-Marc Lachaud, in Bertolt Brecht, sous la direction Philippe Ivernel et Jean-Marc Lachaud, Europe, n 856-857, 2000, p. 159.

16. Initialement, la pièce s'intitulait $L e$ Vol des Lindbergh mais l'aviateur s'étant rallié au nazisme durant la seconde guerre mondiale, Brecht fit disparaitre en 1950 toute référence à son identité, lui substituant une figure anonyme. L'auteur explique ainsi son choix dans un prologue rédigé après la modification de la pièce : «Que son nom soit effacé / Car s'il a trouvé sa voie au-dessus des eaux sans chemins / Il s'est perdu dans le marais de nos villes. [...] / Dix ans / De gloire et de richesse, et puis le misérable / Apprit aux bourreaux hitlériens / À piloter des bombardiers porteurs de mort. Que pour cela / Son nom soit effacé. Mais vous, / Vous voilà mis en garde : ni le courage ni la connaissance / Des moteurs et des cartes marines ne sauraient faire d'un asocial / Un héros d'épopée ». Bertolt Brecht, «Prologue au Vol au-dessus de l'océan " (1950), in Théâtre complet, volume 2, Paris, L'Arche Éditeur, 1974, p. 355.

17. C'est sur le même principe que Brecht écrira, en 1930, deux autres pièces didactiques, Celui qui dit oui Celui qui dit non.

18. Bertolt Brecht, L'Importance d'être d'accord - Pièce didactique de Baden-Baden (1929), traduit par Édouard Pfrimmer et Geneviève Serreau, in Théâtre complet, volume 2, op. cit., pp. 174-175.

19. Philippe Ivernel, «Platon, Kaiser, Brecht - Entre dialogue philosophique, dramatique et épique ", in Dialoguer - Un nouveau partage des voix, actes du colloque de Paris (24-27 mars 2004), sous la direction de Jean-Pierre Sarrazac et Catherine Naugrette, Études théâtrales, nº 31-32, 2005, volume I « Dialogismes », p. 53.

20. Bertolt Brecht, L'Importance d'être d'accord, op. cit., p. 175. 
21. Ibid., pp. 175-176.

22. Ibid., p. 176.

23. Cf. Erwin Piscator, Le Théâtre politique (1929), traduit par Arthur Adamov avec la collaboration de Claude Sebisch, Paris, L’Arche Éditeur, collection « Le Sens de la marche », 1972, pp. 63-69.

24. Sur la relation entre Brecht et Piscator, $c f$. notamment Sylvain Diaz, Philippe Ivernel, Hélène Kuntz, David Lescot, Tania Moguilevskaia, «Mettre en scène l'événement: Tretiakov, Weiss, Brecht, Gatti, Vinaver, Paravidino, Jelinek... ", in La Réinvention du drame (sous l'influence de la scène), sous la direction de Jean-Pierre Sarrazac et Catherine Naugrette, assistés de Ariane Martinez, Julie Valéro et Jonathan Châtel, Études théâtrales, n 38-39, 2007, pp. 82-87.

25. Bertolt Brecht, L'Importance d'être d'accord, op. cit., p. 176.

26. Ibid., pp. 176-177.

27. Ibid., p. 178.

28. Ibid., pp. 179-181.

29. Ibid., p. 181.

30. Id.

31. Bertolt Brecht, L'Achat du cuivre, op. cit., p. 524. [Der Messingkauf, in Werke: Schriften 2, édition établie par Werner Hecht, Jan Knopf, Werner Wittenzwei et Klaus-Detlef Müller, coédition Aufbau-Verlag Berlin und Weimar et Suhrkamp Verlag Frankfurt am Main, 1988, p. 712.]

32. Bertolt Brecht, Le Vol au-dessus de l'océan, (1929), traduit par Gilbert Badia, in Théâtre complet, volume 2, op. cit., pp. 161-163.

33. Bertolt Brecht, L'Importance d'être d'accord, op. cit., p. 173. [Das Badener Lehrstück vom Einverständnis, (1929), in Werke : Stücke 3, op. cit., p. 27.]

34. Bertolt Brecht, La Décision (1931), traduit par Édouard Pfrimmer, in Théâtre complet, volume 2, op. cit.,p. 237. [Die Maßnahme, in Werke : Stücke 3, op. cit., p. 125.]

35. Bertolt Brecht, L'Importance d'être d'accord, op. cit., p. 183.

36. Piscator, Le Théâtre politique, op. cit., p. 67.

37. Cf. Sylvain Diaz, Philippe Ivernel, Hélène Kuntz, David Lescot, Tania Moguilevskaia, « Mettre en scène l'événement », art. cit., p. 85.

38. Bertolt Brecht, L'Importance d'être d'accord, op. cit., p. 183.

39. Ibid., p. 184.

40. Id. [Das Badener Lehrstück vom Einverständnis, op. cit., p. 38.]

41. Id. [Das Badener Lehrstück vom Einverständnis, op. cit., p. 38.]

42. Bertolt Brecht, Le Vol au-dessus de l'océan, op. cit., p. 163.

43. Bertolt Brecht, L'Importance d'être d'accord, op. cit., p. 174.

44. Ibid., p. 176.

45. Bertolt Brecht, « Notes » (1939-1947), in Écrits sur le théâtre [L'Arche], volume II, op. cit., p. 425.

46. Bertolt Brecht, L'Importance d'être d'accord, op. cit., p. 191.

47. Id.

48. Bertolt Brecht, Le Vol au-dessus de l'océan, op. cit., pp. 161-162.

49. Bertolt Brecht, «Le monde d'aujourd'hui peut-il être restitué par le théâtre ? - Contribution écrite aux "Entretiens de Darmstadt" sur le théâtre " (1955), in Écrits sur le théâtre, op. cit., p. 421.

50. Bertolt Brecht, "Représentations fausses de pièces nouvelles » (1951), in Écrits sur le théâtre, op. cit., p. 419.

51. Bertolt Brecht, L'Achat du cuivre, op. cit., p. 686.

52. Philippe Ivernel, « Du spectacle au montage ininterrompu : le Lehrstück brechtien », art. cit., p. 271.

53. Id.

54. Bertolt Brecht, « Du Théâtre quotidien » (1935), in Écrits sur le théâtre, op. cit., p. 669.

55. Bertolt Brecht, «La Scène de la rue - Modèle type d'une scène de théâtre épique » (1938), in Écrits sur le théâtre, op. cit., p. 856. 
56. Cf. notamment Paul Ricœur, Soi-même comme un autre, Paris, Éditions du Seuil, collection « Points / Essais », 1990.

57. Edward Bond, « Note sur une méthode pour le théâtre » (1978), in L'Énergie du sens - Lettres, poèmes et essais (1978-1998), textes édités sous la direction de Jérôme Hankins, Montpellier, Éditions Climats / Maison Antoine Vitez, 2000, p. 136.

58. Cité par David Hirst, Edward Bond, London, Macmillan Publishers, collection «Modern Dramatists ", 1985, p. 126. «Brecht was an experimenter, an explorer. He did not answer all our questions. The time between his death and the present has given us more experience, more history to draw on. The tragedy of twentieth century drama is that Brecht died before he could complete a last series of plays : the plays he would have written as a member and worker of a Marxist society. The loss is very severe. But we have to write the plays he left unwritten. » En l'absence de traduction française du livre de David Hirst, toutes les citations qui en sont extraites sont traduites par mes soins.

59. Edward Bond, Lettre datée du 18 mars 2000 à Rudolf Rach, in La Trame cachée - Notes sur le théâtre et l'État (2000), traduit par Georges Bas, Jérôme Hankins et Séverine Magois, Paris, L'Arche Éditeur, 2003, p. 260.

60. Edward Bond, Café (1995), mise en scène d'Alain Françon, création au Théâtre National de la Colline, Paris, 12 mai 2000.

61. Edward Bond, « Notes sur Café - Pour le Théâtre National de la Colline » (2000), in La Trame cachée, op. cit., pp. 257-258.

62. Edward Bond, "Le Théâtre comme lieu de la justice ", entretien avec Fabienne Darge, Le Monde, 19 avril 2003.

63. Edward Bond, Lettre datée du 18 mars 2000 à Rudolf Rach, op. cit., p. 260.

64. Ibid., p. 264.

65. Ibid., pp. 263-264.

66. Edward Bond, Lettre datée de janvier 1991 à René Loyon citée par Jérôme Hankins, « AvantPropos » aux Poèmes et essais, in Edward Bond, L'Énergie du sens, op. cit., p. 117.

67. Edward Bond, Commentaire sur les Pièces de guerre et le paradoxe de la paix, traduit par Georges Bas, Laure Hémain, Michel Vittoz et Malika B. Durif, Paris, L'Arche Éditeur, 1994, p. 76. [« Drama is not about what happens but about the meanings of what happens. We use values to judge. If an accident happens in real life we may try to establish the facts. But a judge, juror or witness will each have a different version of the accident, based on their social attitude to accidents and all other events. Even non-trivial facts cannot be established without value judgements. Judging is as complex on stage as it is off it. » Commentary on The War Plays, in The War Plays - A Trilogy, London, Methuen Drama, 1991, p. 300.]

68. Edward Bond, Commentaire sur les Pièces de guerre, op. cit., p. 134. [« When people report what it is like to be in a serious accident they say that they were calm and that time seemed to slow so they noticed things they would have had time to notice if they had been strolling by.» Commentary on The War Plays, op. cit., p. 348.]

69. Id. [" Towards the end of Chekhov's Three Sisters the characters on stage talk or fall silent, while off-stage their society - using Solyony as its agent - murders. In our theatre violence is onstage because that is where it is in our life, not waiting in the wings as it was in Chekhov's. His characters hold an inquest on an accident that has not yet taken place but which they cannot prevent. Our stage is the site of the accident. So the scene is faster and more extreme than Chekhov's : it is set in accident-time. Now it is because things happen so rapidly that it is hard to prevent them or escape from them. " Commentary on The War Plays, op. cit., pp. 347-348.]

70. Edward Bond, Lettre du 16 juillet 1997 à Antje Lenkeit, in L'Énergie du sens, op. cit., p. 82.

71. Georges Bas, «Temps-accident », in Edward Bond, La Trame cachée, op. cit., p. 314.

72. Edward Bond désigne ainsi le cycle que constituent quatre de ses pièces les plus récentes qui ont, pour trois d'entre elles, été créées en langue française par Alain Françon alors qu'il était 
directeur du Théâtre National de la Colline à Paris : Café (1995), Le Crime du XXIe siècle (1999), Naître (2006) et People (2006).

73. Pour une analyse approfondie de la production la plus récente d'Edward Bond, $c f$. notamment Sylvain Diaz, "Edward Bond - La Crise épuisée ", in Poétiques de la crise dans les dramaturgies européennes des $\mathrm{XX}^{e}$ et $\mathrm{XXI}^{e}$ siècles, thèse rédigée sous la direction de Jean-Loup Rivière, Université Lumière - Lyon 2, 2009, volume I, pp. 367-447.

74. Nous renvoyons sur cette question de l'héritage brechtien au très beau texte sur lequel Philippe Ivernel et Jean-Marc Lachaud ouvrent le numéro de la revue Europe consacré au dramaturge allemand qu'ils ont dirigé: Philippe Ivernel, Jean-Marc Lachaud, «Brecht aujourd'hui », in Bertolt Brecht, op. cit., pp. 3-7.

INDEX

Mots-clés : accident, Brecht (Bertolt), Lehrstück, pièce didactique 